\title{
LS 5039: A runaway microquasar ejected from the galactic plane
}

\author{
M. Ribó ${ }^{1}$, J. M. Paredes ${ }^{1}$, G. E. Romero ${ }^{2}$, P. Benaglia ${ }^{2}$, J. Martî ${ }^{3}$, O. Fors $^{1}$, and J. García-Sánchez ${ }^{1}$ \\ 1 Departament d'Astronomia i Meteorologia, Universitat de Barcelona, Av. Diagonal 647, 08028 Barcelona, Spain \\ 2 Instituto Argentino de Radioastronomía, C.C.5, (1894) Villa Elisa, Buenos Aires, Argentina \\ 3 Departamento de Física, Escuela Politécnica Superior, Universidad de Jaén, Virgen de la Cabeza 2, 23071 Jaén, \\ Spain
}

Received 10 December 2001 / Accepted 10 January 2002

\begin{abstract}
We have compiled optical and radio astrometric data of the microquasar LS 5039 and derived its proper motion. This, together with the distance and radial velocity of the system, allows us to state that this source is escaping from its own regional standard of rest, with a total systemic velocity of about $150 \mathrm{~km} \mathrm{~s}^{-1}$ and a component perpendicular to the galactic plane larger than $100 \mathrm{~km} \mathrm{~s}^{-1}$. This is probably the result of an acceleration obtained during the supernova event that created the compact object in this binary system. We have computed the trajectory of LS 5039 in the past, and searched for OB associations and supernova remnants in its path. In particular, we have studied the possible association between LS 5039 and the supernova remnant G016.8-01.1, which, despite our efforts, remains dubious. We have also discovered and studied an H I cavity in the ISM, which could have been created by the stellar wind of LS 5039 or by the progenitor of the compact object in the system. Finally, in the symmetric supernova explosion scenario, we estimate that at least $17 M_{\odot}$ were lost in order to produce the high eccentricity observed. Such a mass loss could also explain the observed runaway velocity of the microquasar.
\end{abstract}

Key words. stars: individual: LS 5039, RX J1826.2-1450 - X-rays: binaries - supernovae: individual: G016.8-01.1 - radio continuum: stars - radio continuum: ISM - ISM: supernova remnants

\section{Introduction}

Microquasars are stellar-mass black holes or neutron stars that mimic, on smaller scales, many of the phenomena seen in AGN and quasars. These objects have been found in X-ray binary systems, where a compact object accretes matter from a companion star. Radio emitting X-ray binaries with relativistic radio jets, like SS 433, GRS $1915+105$, GRO J1655-40 or Cygnus X-3, are good examples of microquasars (see Mirabel \& Rodríguez 1999 for a detailed review). With the recent addition of LS 5039 (Paredes et al. 2000), Cygnus X-1 (Stirling et al. 2001) and XTE J1550-564 (Hannikainen et al. 2001) to the microquasar group, the current number of this kind of sources is 14 , among $\sim 280$ known X-ray binaries (Liu et al. 2000; Liu et al. 2001) of which $\sim 50$ display radio emission. Recent studies of microquasars can be found in Castro-Tirado et al. (2001).

Attention to the star LS 5039 was first called by Motch et al. (1997), who proposed it as a High Mass X-ray Binary (HMXB) candidate associated with the X-ray source RX J1826.2-1450. The object is located at an estimated distance of $\sim 3.1 \mathrm{kpc}$ and close to the galactic

Send offprint requests to: M. Ribó,

e-mail: mribo@am.ub.es plane $\left(l=16.88^{\circ}, b=-1.29^{\circ}\right)$. Soon after, non-thermal and moderately variable radio emission was reported by Martí et al. (1998) using the Very Large Array (VLA). The evidence of its microquasar nature was provided by Paredes et al. (2000) when radio jets were discovered with the Very Long Baseline Array (VLBA) at milliarcsecond (mas) angular scales. These authors also pointed out the possible connection of LS 5039 with 3EG J1824-1514, i.e., one of the unidentified EGRET sources of high energy $\gamma$-rays. X-ray observations of RX J1826.2-1450 by Ribó et al. (1999) did not reveal pulsations and were consistent with a significantly hard X-ray spectrum up to $30 \mathrm{keV}$, with a strong Gaussian iron line at $6.6 \mathrm{keV}$.

The mass donor in LS 5039 was originally classified as an O7V((f)) star by Motch et al. (1997). This classification has been recently improved thanks to optical and near infrared spectroscopic observations by Clark et al. (2001), which indicate an $06.5 \mathrm{~V}((\mathrm{f}))$ star.

Our present knowledge of the system orbit is based only on the radial velocity measurements in the optical by McSwain et al. (2001). Their most remarkable findings consist of a short orbital period of $P=4.117$ days and a significant eccentricity of $e=0.41$. They were also able to determine the system radial velocity and a mass function of $f(m)=0.00103 M_{\odot}$. The fact that the available optical 
Table 1. Compilation of optical and radio positions, with associated errors, of LS 5039 .

\begin{tabular}{|c|c|c|c|c|c|c|c|}
\hline $\begin{array}{l}\text { Wavelength } \\
\text { Domain }\end{array}$ & Epoch & $\begin{array}{l}\alpha(\text { ICRS }) \\
(\mathrm{h}, \mathrm{m}, \mathrm{s})\end{array}$ & $\begin{array}{c}\sigma_{\alpha \cos \delta} \\
(\mathrm{mas})\end{array}$ & $\begin{array}{l}\delta(\mathrm{ICRS}) \\
\left({ }^{\circ},{ }^{\prime},{ }^{\prime \prime}\right)\end{array}$ & $\begin{array}{c}\sigma_{\delta} \\
(\mathrm{mas})\end{array}$ & $\begin{array}{l}\text { Catalog } \\
\text { name }\end{array}$ & Reference \\
\hline \multirow[t]{7}{*}{ Optical } & 1905.45 & 182615.0194 & 255 & -145053.300 & 247 & AC 2000.2 & Urban, private communication \\
\hline & 1907.43 & 182615.0177 & 255 & -145053.075 & 247 & AC 2000.2 & Urban, private communication \\
\hline & 1951.577 & 182615.034 & 250 & -145053.59 & 250 & USNO-A2.0 & Monet et al. (1999) \\
\hline & 1979.484 & 182615.0557 & 64 & -145054.075 & 47 & TAC 2.0 & Zacharias \& Zacharias (1999) \\
\hline & 1986.653 & 182615.054 & 300 & -145054.29 & 300 & GSC 1.2 & Morrison et al. (2001) \\
\hline & 1991.75 & 182615.0427 & 149 & -145054.229 & 120 & Tycho-2 & Høg et al. (2000) \\
\hline & 2000.289 & 182615.0563 & 13 & -145054.277 & 13 & UCAC1 & Zacharias et al. (2000) \\
\hline \multirow[t]{2}{*}{ Radio } & 1998.24 & 182615.056 & 10 & -145054.24 & 10 & VLA obs. & Martí et al. (1998) \\
\hline & 2000.42 & 182615.0566 & 4 & -145054.261 & 6 & VLBA obs. & Ribó et al. (2002) \\
\hline
\end{tabular}

photometry does not show an ellipsoidal modulation could indicate that the orbital inclination is very low. Therefore, the possibility of having a black hole in the system cannot be ruled out in spite of the small mass function observed.

The formation of the compact object in an X-ray binary necessarily requires a supernova explosion that does not disrupt the system. This explosive event is expected to considerably change the kinematic properties of the binary star. The change may be rather extreme (i.e., kick velocities approaching $\sim 10^{3} \mathrm{~km} \mathrm{~s}^{-1}$ ) for highly asymmetric supernovae, as it has been proposed for the microquasar Circinus X-1 (Tauris et al. 1999). This mechanism may also be responsible for ejecting X-ray binaries into the halo of the Galaxy. The fast moving X-ray nova XTE J1118+480 is a possible example (Mirabel et al. 2001), although it could have been ejected from a globular cluster in the past. On the other hand, the microquasars GRO J1655-40 and Cygnus X-1, also display runaway velocities (Shahbaz et al. 1999; Kaper et al. 1999). With independence of their origin in the galactic plane or in the halo, the existence of runaway microquasars is a new and important issue that deserves an in-depth study.

In this paper we focus on the kinematic properties of LS 5039 and its surroundings. In Sect. 2 we estimate the proper motions of the system, using optical and radio data. In Sect. 3 we discuss the distance and space velocity of LS 5039, whereas in Sect. 4 we analyze the trajectory of the binary system in the past. In Sect. 5 we study the possible association of this microquasar with a supernova remnant, and in Sect. 6 we analyze their H I surroundings. Finally, we make a global discussion in Sect. 7 and summarize our main conclusions in Sect. 8.

\section{Positions and proper motions}

\subsection{Optical positions}

LS $5039(V \simeq 11.2, B \simeq 12.2)$ is not included in most accurate astrometric catalogs, like Hipparcos and Tycho-1 (which are complete up to $V \sim 9.0$ and $V \sim 10.0$, respectively). Although it appears in Tycho-2, its position has a large uncertainty when compared to the average error within its magnitude range (Høg et al. 2000). As a result, the catalogued proper motions lack of the required precision. Moreover, other astrometric catalogs which were not included in Tycho-2 estimate of the proper motions, have been released since then. In view of all these facts, we decided to carry out a thorough study of all available catalogs. First, we did a search using the query forms within the VizieR database (Ochsenbein et al. 2000) at the Centre de Données astronomiques de Strasbourg (CDS), and afterwards we looked for new information on several public web sites. The results of this search are listed in Table 1, with all coordinates in ICRS, and commented below.

The oldest astrometric information for LS 5039 is dated around 1905 and comes from the Astrographic Catalog 2000 (AC 2000, Urban et al. 1998). However, a re-reduction of this catalog was performed recently, using an improved reference catalog that allowed a better handling of the systematic errors (S. E. Urban, private communication). This new catalog will soon be released as AC 2000.2. Since changes were most significant in the faint stars of the southern AC 2000 zones, the position of LS 5039 suffered a noticeable shift. Actually, two positions corresponding to different epochs are given in AC 2000.2.

The following available astrometry, dated around 1950, comes from the scanning and reduction of the Palomar Observatory Sky Survey plates, carried out by Monet et al. (1999), which is part of the USNO-A2.0 catalog.

We inspected the Second Cape Photographic Catalog (CPC2, Zacharias et al. 1999), a Southern Hemisphere astrometric catalog, containing observations between 1962 and 1973. Unfortunately, LS 5039 is not listed in it, because the limiting magnitude of this catalog was $V \simeq 10.5$.

The Twin Astrographic Catalog version 2 (TAC 2.0) provides positional information around 1980. Apart from the internal error of $\sigma_{\alpha \cos \delta}=62$ mas and $\sigma_{\delta}=45$ mas associated to the LS 5039 position, an external error of 15 mas has been taken into account following an estimate carried out by Zacharias \& Zacharias (1999).

The last photographic catalog used is the Guide Star Catalog version 1.2 (GSC 1.2, Morrison et al. 2001). This is a re-reduction of the GSC (Lasker et al. 1990), after removing plate-based systematic distorsions that are a function of magnitude and radial distance from the plate 
center. In addition, by using the Starlink library SLALIB, we have transformed the catalogued position from FK5 to ICRS.

As mentioned above, LS 5039 appears as well in the Tycho-2 catalog (Høg et al. 2000). The average internal error in position for a $V \simeq 11.2$ star is around 50 mas, while the errors in $\alpha \cos \delta$ and $\delta$ for LS 5039 are 93 and 120 mas, respectively. This fact tells us that the position estimate is not as good as one would expect. On the other hand, the scatter-based errors from the 4 positions present in the Tycho-2 data are 149 and 60 mas, respectively. Hence, we have considered $\sigma_{\alpha \cos \delta}=149$ mas and $\sigma_{\delta}=120$ mas.

A second epoch of the GSC was performed during the nineties. However, the positional errors in the current version of this catalog, GSC 2.2.01, are intended as indicators for operational use only, and cannot be used for scientific studies. Hence, we have considered that this catalog does not provide accurate astrometric information to be used for estimating proper motions.

Finally, we have used the first release of the US Naval Observatory CCD Astrograph Catalog (UCAC1, Zacharias et al. 2000). As can be seen in Table 1, this is by far the best optical astrometric catalog available.

\subsection{Radio positions}

Independent astrometric information can be obtained from radio observations of LS 5039. The first available astrometric information comes from the NVSS by Condon et al. (1998). However, the position error in this catalog, around $1^{\prime \prime}$, is too large to be of any use for our purposes. Martí et al. (1998) carried out $\mathrm{VLA}^{1}$-A configuration observations which provided accurate (10 mas) astrometry for LS 5039. Finally, phase-referencing VLBA ${ }^{1}$ observations conducted by Ribó et al. (2002) provide the last radio position, with the best accuracy among all available data. Details of the astrometry for the last two cases are listed in Table 1. The relatively large location error of few mas in the VLBA data is due to confusion produced by the high level of Galactic electron scattering in this region (for both the reference source and LS 5039).

We must state that no correction for parallax has been applied to the obtained positions, since at an estimated distance of $\sim 3 \mathrm{kpc}$, this would translate into less than half a mas, which is always much smaller than the available uncertainties.

\subsection{Proper motions}

It is clear from the astrometric data that both, the optical and radio sources, are almost in the same position of the sky. However, in order to show that this is not a chance coincidence and that both emissions originate in the same

\footnotetext{
1 The VLA and the VLBA are operated by the National Radio Astronomy Observatory (NRAO). The NRAO is a facility of the National Science Foundation operated under cooperative agreement by Associated Universities, Inc.
}

Table 2. Proper motions estimates for LS 5039.

\begin{tabular}{lcr}
\hline Data set & $\begin{array}{c}\mu_{\alpha \cos \delta} \\
\left(\mathrm{mas} \mathrm{yr}^{-1}\right)\end{array}$ & $\begin{array}{c}\mu_{\delta} \\
\left(\mathrm{mas} \mathrm{yr}^{-1}\right)\end{array}$ \\
\hline Optical & $4.7 \pm 1.3$ & $-11.0 \pm 0.8$ \\
Radio & $4.0 \pm 4.9$ & $-9.6 \pm 5.3$ \\
Optical+Radio & $4.7 \pm 1.1$ & $-10.6 \pm 1.0$ \\
\hline
\end{tabular}

object, we have computed independent proper motions for the optical and radio data.

Although the position uncertainties from the AC 2000.2 and USNO-A2.0 catalogs are relatively large, the epoch span achieved justifies their inclusion in the estimate of the proper motions. Hence, from the optical data, and taking into account the astrometric uncertainties, we obtain the following results:

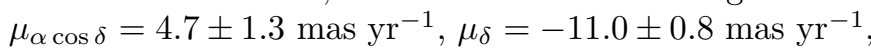
where the errors come directly from the least squares fit. The two accurate radio positions give proper motions of:

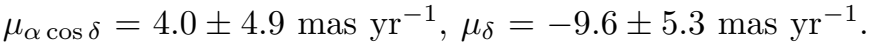
Although this last result has a large error because only two points are available, we can say that both, the optical and radio sources, have very similar proper motions. Therefore, based only on astrometric data, we are able to confirm that both, the optical and the radio emission, originate in the same object. Hence, we will use all the data listed in Table 1 to compute accurate proper motions for LS 5039, which happen to be:

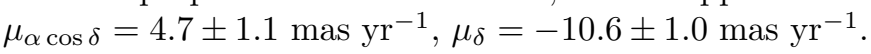
All these results are summarized in Table 2. We can now transform the proper motions into galactic coordinates and obtain $\mu_{l}=-7.2 \pm 1.0$ mas $\mathrm{yr}^{-1}$ and $\mu_{b}=-9.1 \pm 1.0$ mas $\mathrm{yr}^{-1}$. It is clear from these results that there is a noticeable motion perpendicular to the galactic plane and moving away from it.

According to our least squares fits, and defining $t=$ yr -2000.0 , the predicted ICRS values for $\alpha$ and $\delta$ near $t=0$ are:

$$
\begin{aligned}
& \alpha=18^{\mathrm{h}} 26^{\mathrm{m}} 15.0565^{\mathrm{s}}+\left[\frac{4.7 t / \cos \delta}{\operatorname{mas}}\right] \\
& \delta=-14^{\circ} 50^{\prime} 54.260^{\prime \prime}-\left[\frac{10.6 t}{\operatorname{mas}}\right]
\end{aligned}
$$

being $\sqrt{9+1.2 t^{2} / \cos ^{2} \delta}$ and $\sqrt{9+t^{2}}$ the errors in mas in $\alpha$ and $\delta$, respectively. Comparing the fits with the positions in Table 1 and the proper motions in Table 2 we can say that, approximately, the offsets are determined by the VLBA data point because of the small error in position, while the proper motions are obtained by the optical points due to the huge time span.

In Fig. 1 we have plotted the offsets in $\alpha \cos \delta$ and $\delta$ from the fitted values for year 2000.0 versus time, for the data listed in Table 1, together with the respective fits to the data. Notice that in both plots the UCAC1 and VLBA data are almost superimposed. 

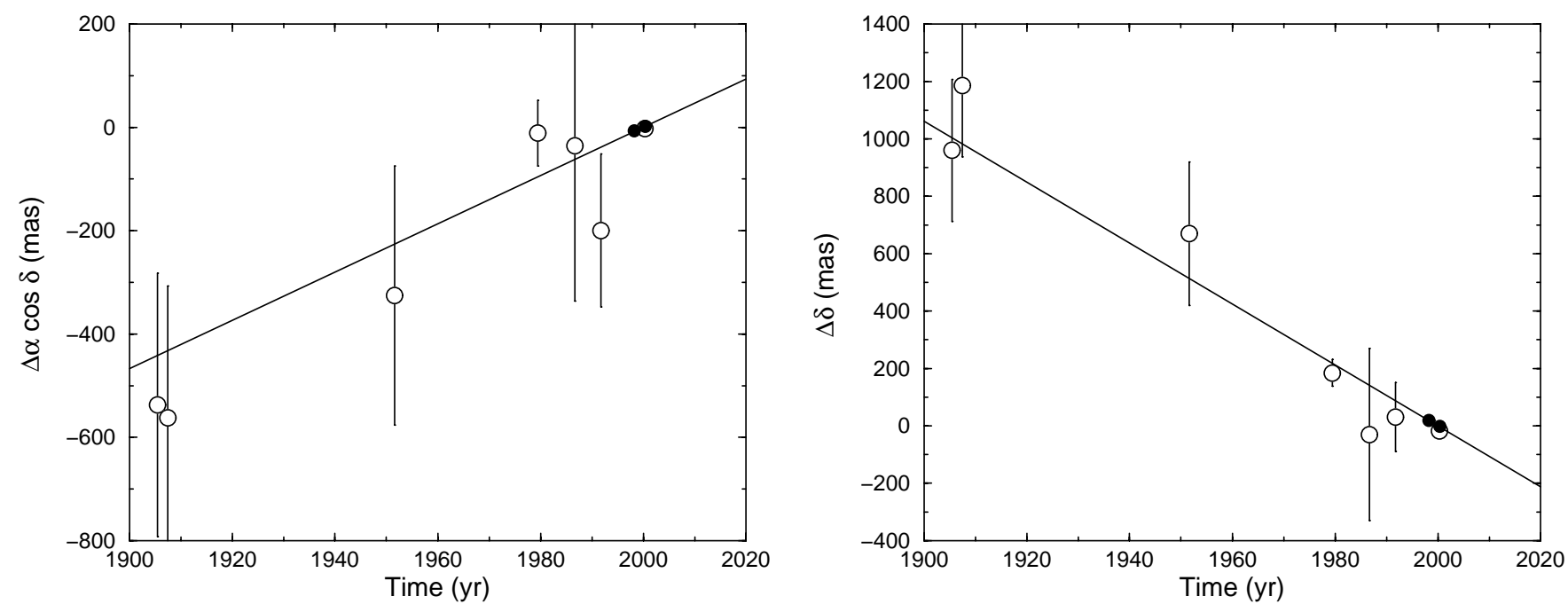

Fig. 1. Offsets in Right Ascension (left) and Declination (right), relatives to the fitted ones for year 2000.0, versus time for all the positions listed in Table 1. Open circles represent the optical positions, and filled circles the radio ones. The solid lines represent the least squares fits to the whole data sets.

Table 3. Distance estimates to LS 5039 derived from optical photometry.

\begin{tabular}{ccc}
\hline$V$ & $B-V$ & $d(\mathrm{kpc})$ \\
\hline $11.23 \pm 0.01^{a}$ & $0.95 \pm 0.01^{a}$ & $2.68 \pm 0.06$ \\
$11.20 \pm 0.02^{b}$ & $0.95 \pm 0.02^{b}$ & $2.64 \pm 0.06$ \\
$11.33 \pm 0.02^{c}$ & $0.85 \pm 0.02^{c}$ & $3.26 \pm 0.07$ \\
$11.32 \pm 0.01^{d}$ & $0.85 \pm 0.02^{d}$ & $3.25 \pm 0.07$ \\
\hline
\end{tabular}

${ }^{a}$ Drilling (1991).

${ }^{b}$ Lahulla \& Hilton (1992).

${ }^{c} 1996$ October observations by Clark et al. (2001).

${ }^{d} 2000$ September observations by Clark et al. (2001).

\section{Ejection from the galactic plane}

Since the distance to LS 5039 and its uncertainty are fundamental to compute the space velocity of the system, we think it is worth to perform first of all a short study on this issue.

\subsection{Distance and its uncertainty}

Motch et al. (1997) proposed a distance of $3.1 \mathrm{kpc}$ to LS 5039 based on the star color excess, and stated that this estimate could have a large uncertainty. This value was computed assuming an $\mathrm{O} 7 \mathrm{~V}((\mathrm{f}))$ spectral type. However, recent observations by Clark et al. (2001) show that the optical companion has a spectral type of $\mathrm{O} 6.5 \mathrm{~V}((\mathrm{f}))$. On the other hand, old calibrations for the intrinsic color index and absolute magnitude (Johnson 1966; Deutschman et al. 1976) were used by Motch et al. (1997). Hence, we have performed a new estimate of the distance taking into account the new spectral type and more recent calibrations.

The optical photometry available up to now, containing at least $B$ and $V$ magnitudes comes from
Drilling (1991), Lahulla \& Hilton (1992) and Clark et al. (2001), and is listed in Table 3. Using an intrinsic color index of $(B-V)_{0}=-0.30 \pm 0.02$ for an $06.5 \mathrm{~V}$ star (Schaerer et al. 1996; Lejeune \& Schaerer 2001), we can compute the color excess $E(B-V)$ for all the observations. Finally, using the relationship $A_{V}=(3.30+0.28(B-$ $\left.V)_{0}+0.04 E_{B-V}\right) E(B-V)$ (Schmidt-Kaler 1982) and $M_{V}=-4.99 \pm 0.3$ for an $06.5 \mathrm{~V}$ star (Vacca et al. 1996) we obtain the distance estimates listed in Table 3 . The weighted mean of these values is $2.9 \pm 0.3 \mathrm{kpc}$, and will be the distance to LS 5039 used hereafter. Finally, assuming that the Sun is at $8.5 \mathrm{kpc}$ from the galactic center, we obtain a galactocentric distance of $5.8 \pm 0.3 \mathrm{kpc}$ for LS 5039.

\subsection{Space velocity}

If we assume a distance of $2.9 \pm 0.3 \mathrm{kpc}$ to $\mathrm{LS} 5039$, as seen in the previous section, and a systemic $V_{\mathrm{r}}=$ $4.6 \pm 0.5 \mathrm{~km} \mathrm{~s}^{-1}$ (McSwain et al. 2001), the proper motions estimates translate into $(U=40 \pm 5, V=-82 \pm 16$, $W=-118 \pm 19) \mathrm{km} \mathrm{s}^{-1}$ in the Local Standard of Rest $($ LSR $)$ defined by $\left(U_{\odot}=9, V_{\odot}=12, W_{\odot}=7\right) \mathrm{km} \mathrm{s}^{-1}$. This gives a total systemic velocity of $v_{\mathrm{sys}}=\left(U^{2}+V^{2}+\right.$ $\left.W^{2}\right)^{1 / 2}=149 \pm 18 \mathrm{~km} \mathrm{~s}^{-1}$. Using a value of $215 \mathrm{~km} \mathrm{~s}^{-1}$ for the galactic rotation at $5.8 \mathrm{kpc}$ from the galactic center (Fich et al. 1989) we can transform the galactic plane space velocities $(U$ and $V$ ) from the LSR into the LS 5039 regional standard of rest (RSR). Applying this transformation we find $U=51 \pm 6$ and $V=-71 \pm 16 \mathrm{~km} \mathrm{~s}^{-1}$, and $v_{\text {sys }}=147 \pm 17 \mathrm{~km} \mathrm{~s}^{-1}$. All these results are summarized in Table 4 .

Taking into account that the cosmic dispersion for early-type stars is $\left(\sigma_{U}, \sigma_{V}, \sigma_{W}\right) \simeq(7,8,4) \mathrm{km} \mathrm{s}^{-1}$ (Torra et al. 2000) we can conclude that this source is escaping from its own RSR and has a large velocity component perpendicular to the galactic plane. Since LS 5039 is a HMXB 
Table 4. Space velocity estimates, in $\mathrm{km} \mathrm{s}^{-1}$, for LS 5039 related to the LSR and to its RSR.

\begin{tabular}{lcccc}
\hline Frame & $U$ & $V$ & $W$ & $v_{\text {sys }}$ \\
\hline LSR & $40 \pm 5$ & $-82 \pm 16$ & $-118 \pm 19$ & $149 \pm 18$ \\
RSR & $51 \pm 6$ & $-71 \pm 16$ & $-118 \pm 19$ & $147 \pm 17$ \\
\hline
\end{tabular}

containing an early-type star, it seems very unlikely to be a high speed halo object crossing the galactic plane. Hence, the most plausible explanation for the observed velocity is that the binary system obtained an acceleration during the supernova event that created the compact object in this microquasar.

\section{The past trajectory of LS $\mathbf{5 0 3 9}$}

An interesting thing to do once a position and space velocity estimates are available, is to compute the trajectory of LS 5039 in the past, and then to look for OB associations and SN Remnants (SNRs) in its path, in order to establish possible relationships. For this purpose, we computed the galactic orbital motion of this system under the gravitational field of the Galaxy. The galactic mass model by Dauphole \& Colin (1995) was adopted for the integration, which was was performed using a Runge-Kutta fourth-order integrator using time steps of 1000 years.

It is helpful to search for $\mathrm{OB}$ associations in the past trajectory of runaway X-ray binaries, as done by Ankay et al. (2001) with HD 153919/4U 1700-37, because if evidences are found that the runaway system originated in an $\mathrm{OB}$ association, it is possible to estimate the age of the binary system after the SN explosion. According to the computed trajectory in the past for LS 5039, there are two $\mathrm{OB}$ associations in or close to its path in the plane of the sky: Sct OB3 and Ser OB2 (Melnik \& Efremov 1997). Unfortunately, the distance to Sct OB3 is $\sim 1.5 \mathrm{kpc}$ (Melnik \& Efremov 1997), and the distance to Ser OB2 is $1.9 \pm 0.3 \mathrm{kpc}$ (Forbes 2000). Hence, the two OB associations found in the path of LS 5039 are too close to us to be related to it.

Since we have not been able to fix a limit on the integration time in the past thanks to a possible relationship with an OB association, we will use the vertical distribution of early-type stars in the Galaxy for this purpose. O-type stars are typically located at distances within $45 \pm 20 \mathrm{pc}$ from the galactic midplane (Reed 2000). According to the galactic latitude of LS 5039 and using a distance of $2.9 \pm 0.3 \mathrm{kpc}$, its actual height is $Z=-65 \pm 7$ pc. Since it is escaping from the galactic plane, we can compute the trajectory backward in time up to when it had a height of $Z=65 \mathrm{pc}$, which is $\sim 1.1 \mathrm{Myr}$ ago. Hence, the SN explosion probably took place in the last $\sim 1.1 \mathrm{Myr}$.

Thus, for a possible association of LS 5039 with a SNR, we will focus our attention on the galactic trajectory of the binary system for the last $\sim 1$ Myr. In addition, the likelihood of detecting a SNR decreases with time, so it is

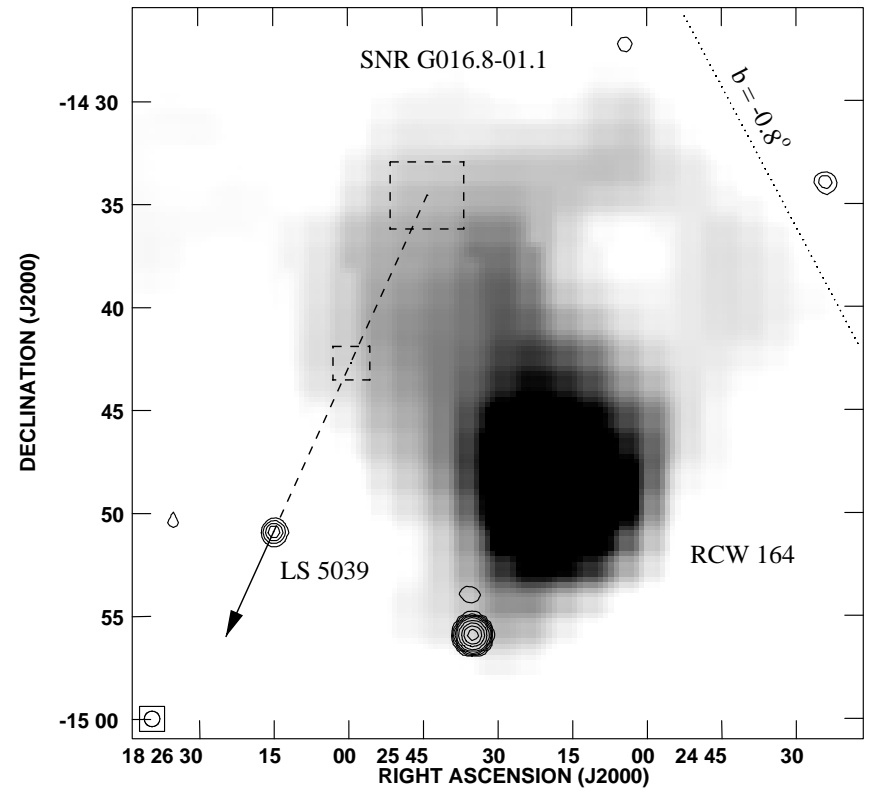

Fig. 2. Wide field radio map of LS 5039, its nearby shell-like SNR G016.8-01.1 and the HiI-region RCW 164 (which is the stronger source in the field). The grey scale emission is taken from the Parkes-MIT-NRAO tropical survey at the $6 \mathrm{~cm}$ wavelength (Tasker et al. 1994). The overlaid contours correspond to the NVSS map of the region at the $20 \mathrm{~cm}$ wavelength (Condon et al. 1998). The arrow marks the proper motion sense (see text). The dashed line is the computed trajectory for the last $10^{5} \mathrm{yr}$, with the corresponding error boxes in position at that epoch and $5 \times 10^{4}$ yr ago. The dotted line represents positions with a galactic latitude of $-0.8^{\circ}$ for reference purposes.

not expected that the radio emission of the SNR can be observed much beyond the time interval considered above (e.g., Shklovskii 1968).

A search in a catalog of galactic supernova remnants (Green 2000) reveals the presence of several SNRs near the path of LS 5039 on the plane of the sky, though the center of none of them is crossed by its trajectory. Only three of them, namely SNR G016.7+00.1, SNR G16.8-01.1, and SNR G018.8+00.3, are within $1^{\circ}$ of the LS 5039 path. The distance to SNR G016.7+00.1 was estimated to be at $\sim 14$ kpc by Reynoso \& Mangum (2000), making impossible an association with LS 5039. Dubner et al. (1999) concluded that SNR G018.8+00.3 is located at $1.9 \pm 0.5 \mathrm{kpc}$, and has an age of $\sim 16000 \mathrm{yr}$. Although the distance is not very different from the one to LS 5039, the age of this SNR is by far too short to be associated with the microquasar. Hence, the only remaining candidate is SNR G16.8-01.1, which deserves a detailed study.

\section{SNR G016.8-01.1}

A wide field radio map of the surrounding of LS 5039 is shown in Fig. 2, with the microquasar (contours) and the nearby SNR G016.8-01.1 (grey scale), together with the position of the H II-region RCW 164 (which is the stronger source in the field). The arrow in this figure marks the proper motion sense of LS 5039 as it would be seen from 
the LSR (i.e., correction for the peculiar velocity of the Sun has been applied), while the dashed line represents the trajectory in the past up to $10^{5} \mathrm{yr}$, with the corresponding error boxes in position at that epoch and $5 \times 10^{4} \mathrm{yr}$ ago. From this figure we can see that LS 5039 crosses the projection in the plane of the sky of SNR G016.8-01.1 between $\sim 4 \times 10^{4}$ and $\sim 1.3 \times 10^{5}$ years ago.

If one of the two stars forming a close binary system experiences a SN explosion, it may form a SNR and an $\mathrm{X}$-ray binary. Since conservation of the linear momentum after the SN explosion is required, if the SNR and the $\mathrm{X}$-ray binary are related, we expect them to be aligned with the proper motion of the latter, and any deviation from this behavior should be explained by the projection in the plane of the sky of the initial space velocity of the binary system prior to the SN event. This does not seem to be the case here, because SNR G016.8-01.1 and LS 5039 are not well aligned with the LS 5039 proper motion. Moreover, if we assume that the center of the shelllike remnant is located at the minimum of radio emission in Fig. 2, approximately located at $\alpha=18^{\mathrm{h}} 25^{\mathrm{m}} 03^{\mathrm{s}}$, $\delta=-14^{\circ} 37^{\prime} 30^{\prime \prime}\left(\right.$ or $\left.l=16.94^{\circ}, b=-0.93^{\circ}\right)$, a peculiar velocity in the plane of the sky for the original system of $\simeq 70 \mathrm{~km} \mathrm{~s}^{-1}$ is obtained. This velocity is much higher than the typical $\sim 10 \mathrm{~km} \mathrm{~s}^{-1}$ found for early-type stars. In order to reduce this velocity we can use in our favor the error in the proper motion angle, and find that it turns out to be $\simeq 50 \mathrm{~km} \mathrm{~s}^{-1}$, which is still too high. However, the center of the shell-like remnant could be in another position, closer to the LS 5039 trajectory, and the derived peculiar velocity for the system prior to the SN explosion could fit in the typical values of early-type stars. Hence, from the kinematical point of view, we are not able to firmly discard a possible association between both objects. Therefore, we have performed an in-depth study of the remnant.

SNR G016.8-01.1 was discovered by Reich et al. (1986) using a combination of survey data analysis and new multifrequency observations. The extended radio source has a complex structure due to the superposition of the HiI-region RCW 164 (Rodgers et al. 1960), which is coincident with the peak of the continuum emission (in black in Fig. 2). The diffuse radiation observed around the peak is strongly polarized (Rodgers et al. 1960), indicating a synchrotron nature. Polarization decreases towards the position of the foreground HiI-region as a result of the thermal contribution. Thermal and non-thermal components of the radiation cannot be clearly separated, but the total flux from the SNR at $5 \mathrm{GHz}$ seems to be $\sim 1 \mathrm{Jy}$ (J. A. Combi, private communication). The angular diameter of the remnant is $\sim 30^{\prime}$; its distance remains unknown.

\subsection{A lower limit of the distance}

In order to determine a lower limit of the distance to SNR G16.8-1.10, we carried out $\mathrm{H} 166 \alpha$ recombination line $(1424.734 \mathrm{MHz})$ observations of the foreground HiI-region in November 17th 2000. We used a 30-m

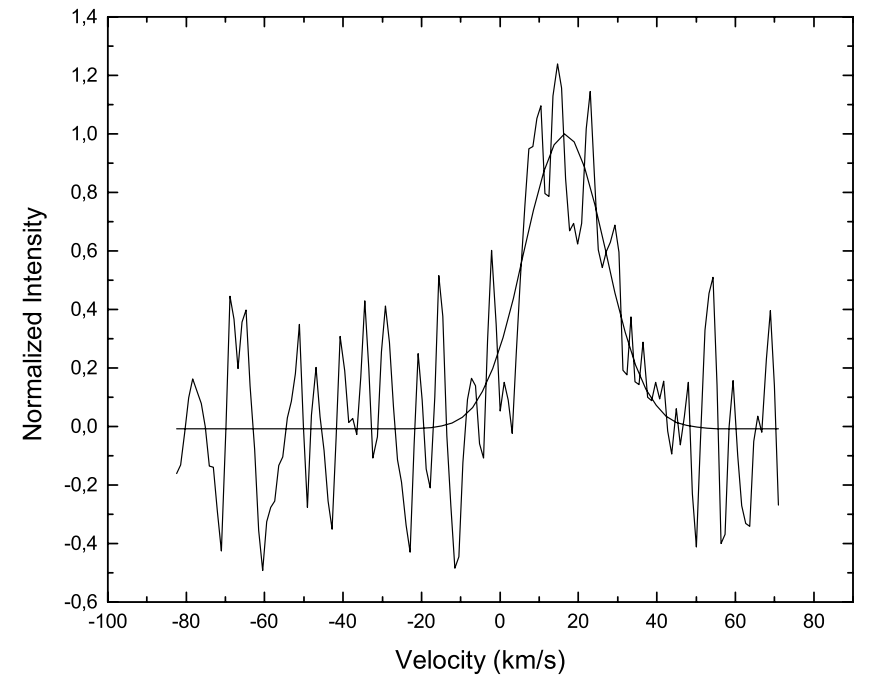

Fig. 3. H166 $\alpha$ line observations of the HiI-region RCW 164. The solid line represents a Gaussian fit to the data, with its maximum being at $v=16.5 \pm 0.8 \mathrm{~km} \mathrm{~s}^{-1}$.

radiotelescope at the Instituto Argentino de Radioastronomía (IAR), Villa Elisa. The receiver is a helium-cooled HEMT amplifier with a 1008-channel autocorrelator at the back end. The HPBW at a wavelength of $21 \mathrm{~cm}$ is $30^{\prime}$ and the temperature of the system on the cold sky during the observations was about $35 \mathrm{~K}$. The $\mathrm{H} 166 \alpha$ line was detected at a velocity of $16.5 \pm 0.8 \mathrm{~km} \mathrm{~s}^{-1}$ (see Fig. 3) after 1.5 hours of integration time, with a signal-to-noise ratio of $\sim 4$. At a location of $l \approx 16.8^{\circ}$, $b \approx-1.1^{\circ}$, standard galactic rotation models (Fich et al. 1989) indicate that the observed velocity corresponds to a distance $d \sim 1.8 \mathrm{kpc}$. Consequently, SNR G16.8-1.10 should be farther than $\sim 2 \mathrm{kpc}$, as suggested by its relatively small angular size.

\subsection{Particle density estimates}

If we adopt for SNR G016.8-01.1 the same distance to LS 5039, we can make some estimates of the parameters that would characterize the SNR in case of being associated with the X-ray binary. Using a size of $30^{\prime}$ and a distance of $2.9 \mathrm{kpc}$, the inferred radius would be $R=12.7 \mathrm{pc}$ and it would be, thus, still in the adiabatic expansion phase. Using the standard Sedov (1959) solutions, we can express the particle density of the ambient medium as

$n=4.44 \times 10^{-8} t^{2} E_{51} R_{1}^{-5} \mathrm{~cm}^{-3}$,

where $t$ is the age of the remnant in years, $E_{51}$ is the original energy release of the SN explosion in units of $10^{51} \mathrm{erg}$ and $R_{1}$ is the remnant radius in units of $10 \mathrm{pc}$. Since the binary system is bound after the SN explosion, its total mass has to be larger than the mass of the SNR (van den Heuvel 1978), which, therefore, should be moving at least with the same space velocity in the opposite direction. Hence, the maximum age of the remnant if it were related to LS 5039, would be around $5 \times 10^{4} \mathrm{yr}$. If we use this age and assume that $E_{51}=0.4$ (Spitzer 1998), we find an ambient density 
of $n \sim 13.5 \mathrm{~cm}^{-3}$. Considering the uncertainty in the distance, we can say that $8<n<23 \mathrm{~cm}^{-3}$. Although such number densities would not be very unusual towards this direction of the inner part of the Galaxy, we have made $\mathrm{H}$ I observations in order to estimate the typical column densities in the region of interest.

The H I observations were performed during November 29th and December 1st 2000, with the already mentioned IAR telescope. The Hi line was observed in a hybrid total-power mode with a sampling on a $0.25^{\circ}$-lattice around the position of SNR G016.8-01.1, covering a total of $4^{\circ} \times 4^{\circ}$. The velocity resolution obtained was $1.05 \mathrm{~km} \mathrm{~s}^{-1}$, with a total coverage of $\pm 450 \mathrm{~km} \mathrm{~s}^{-1}$. The rms level in brightness temperature was $\sim 0.1 \mathrm{~K}$. Those channel maps in the velocity range from 10 to $50 \mathrm{~km} \mathrm{~s}^{-1}$ (corresponding to distances between 1.5 and $4.5 \mathrm{kpc}$ ) were inspected looking for clouds or a local minimum that could be associated to the SNR.

The maps show a strong gradient of brightness temperature towards the galactic plane, but no clear evidence for a structure that could be associated with the SNR. In Fig. 4, we show the integrated column density map for the velocity interval between $26-34 \mathrm{~km} \mathrm{~s}^{-1}$, where the contour labels are in units of $10^{19} \mathrm{~cm}^{-2}$. With the column densities observed in the region where SNR G016.8-01.1 is located $\left(\sim 4 \times 10^{20} \mathrm{~cm}^{-2}\right)$, the ambient density should be $\sim 5 \mathrm{~cm}^{-3}$. This is nearly a factor of 3 lower than the estimated density from the expected size of the SNR if it is at $2.9 \mathrm{kpc}$. Taking into account the uncertainty in the distance, the ambient density determined from the remnant's size $\left(8<n<23 \mathrm{~cm}^{-3}\right)$ would be slightly larger than that estimated from the $\mathrm{H}$ i observations. It could be that there is more material under the form of other molecular species, or that the original SN energy release could have been different from what we have assumed, or, finally, that the distance to the SNR is not $2.9 \mathrm{kpc}$ and it is not related to LS 5039. The case, consequently, remains unsolved.

\section{The $H$ I surroundings of LS 5039}

Notwithstanding the absence of structures that can be clearly associated with the SNR in the $\mathrm{H}$ I distribution, the channel maps in the interval $10-46 \mathrm{~km} \mathrm{~s}^{-1}$ (see Fig. 5) reveal the existence of a large, semi-open cavity which is very similar to bubbles blown out by early-type stars in regions with steep density gradients (e.g., Benaglia \& Cappa 1999). This phenomenon has also been observed around another HMXB (HD 153919) by Benaglia \& Cappa (1999). The ambient material is thought to be swept up by the strong wind of the massive early-type star in the binary system creating a local minimum around the star. Density gradients towards the galactic plane in the original matter distribution can result in a preferred escape direction for the wind, yielding open structures. Even in some cases the star appears displaced from the actual minimum in the $\mathrm{H}$ I distribution.

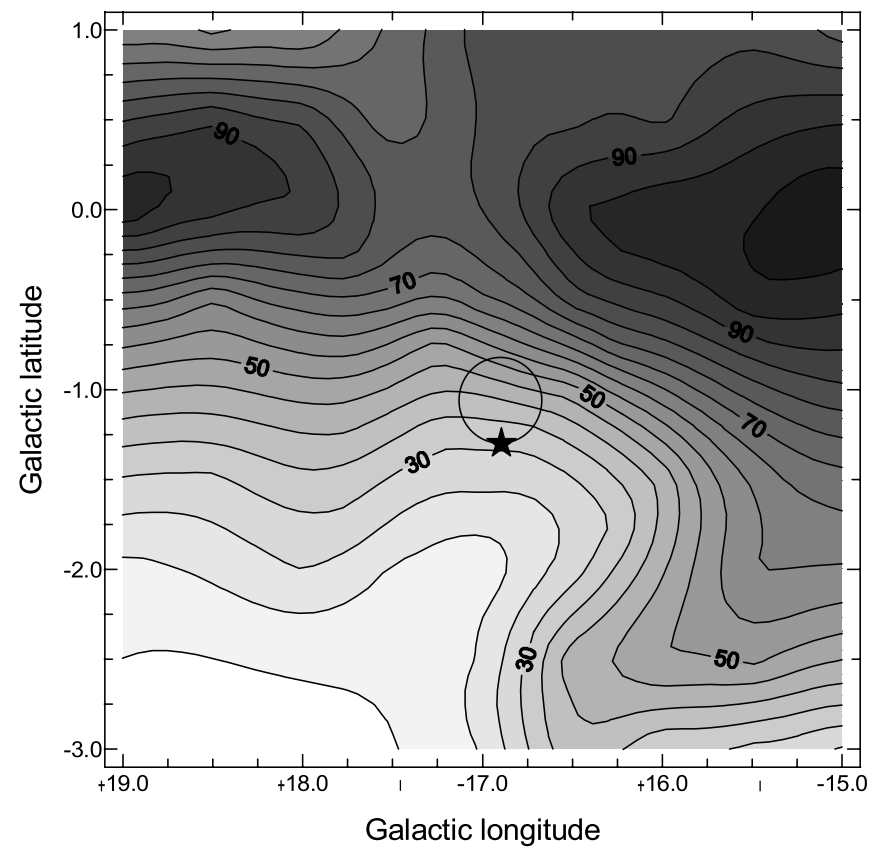

Fig. 4. Neutral hydrogen column density distribution towards SNR G016.8-01.1, integrated over the velocity interval $26-34 \mathrm{~km} \mathrm{~s}^{-1}$. Contour levels are in units of $10^{19} \mathrm{~cm}^{-2}$. The circle marks the position of SNR G016.8-01.1, while the star represents LS 5039.

The bubble detected around LS 5039 is centered at a systemic velocity of $v_{\text {sys }} \simeq 33 \mathrm{~km} \mathrm{~s}^{-1}$, with an expansion velocity $v_{\exp } \simeq 12 \mathrm{~km} \mathrm{~s}^{-1}$. The galactic rotation model by Fich et al. (1989) indicates that it is located at a distance of $\simeq 3.2 \mathrm{kpc}$, quite consistent with the distance to the microquasar. The total mass removed in order to create the cavity is $\sim 1.5 \times 10^{4} M_{\odot}$, implying a kinetic energy $\sim 2 \times 10^{49} \mathrm{erg} \mathrm{s}^{-1}$, which can be considered as a rough estimate of the energy deposited by the wind of the star into the ISM. Such a value is in accordance with previous estimates for similar Of star wind blown bubbles (e.g., Benaglia \& Cappa 1999). With a radius of $\sim 60 \mathrm{pc}$, the dynamical age of the bubble is $\sim 3 \times 10^{6} \mathrm{yr}$, which should be considered as an upper limit for its actual age since it ignores possible contributions from the $\mathrm{SN}$ explosion and the wind of the progenitor of the compact star.

We have also searched for other contributors to the cavity, among stars earlier than B2 that were projected over the cavity, and whose distances were in agreement with that of LS 5039. It seems unlikely that the Wolf Rayet star WR 115 (WN6+OB?) could contribute in some way, because its distance is $2 \mathrm{kpc}$, and is located over the shell of the cavity $\left(l=16.98^{\circ}, b=-1.03^{\circ}\right)$. The other luminous stars in the field, LS 5005, LS 5017, LS 5022, LS 5047, LS 5048 and HD 169673 can sum up at most $20 \%$ of the energy required to create such a minimum in the $\mathrm{HI}$ distribution. The $\mathrm{O}((\mathrm{f}))$ star in LS 5039, then, seems to be the main agent forming the bubble.

However, the actual space velocity indicates that the system has been close to the cavity only during the last $\sim 2 \times 10^{5} \mathrm{yr}$, which is much shorter than the upper limit 

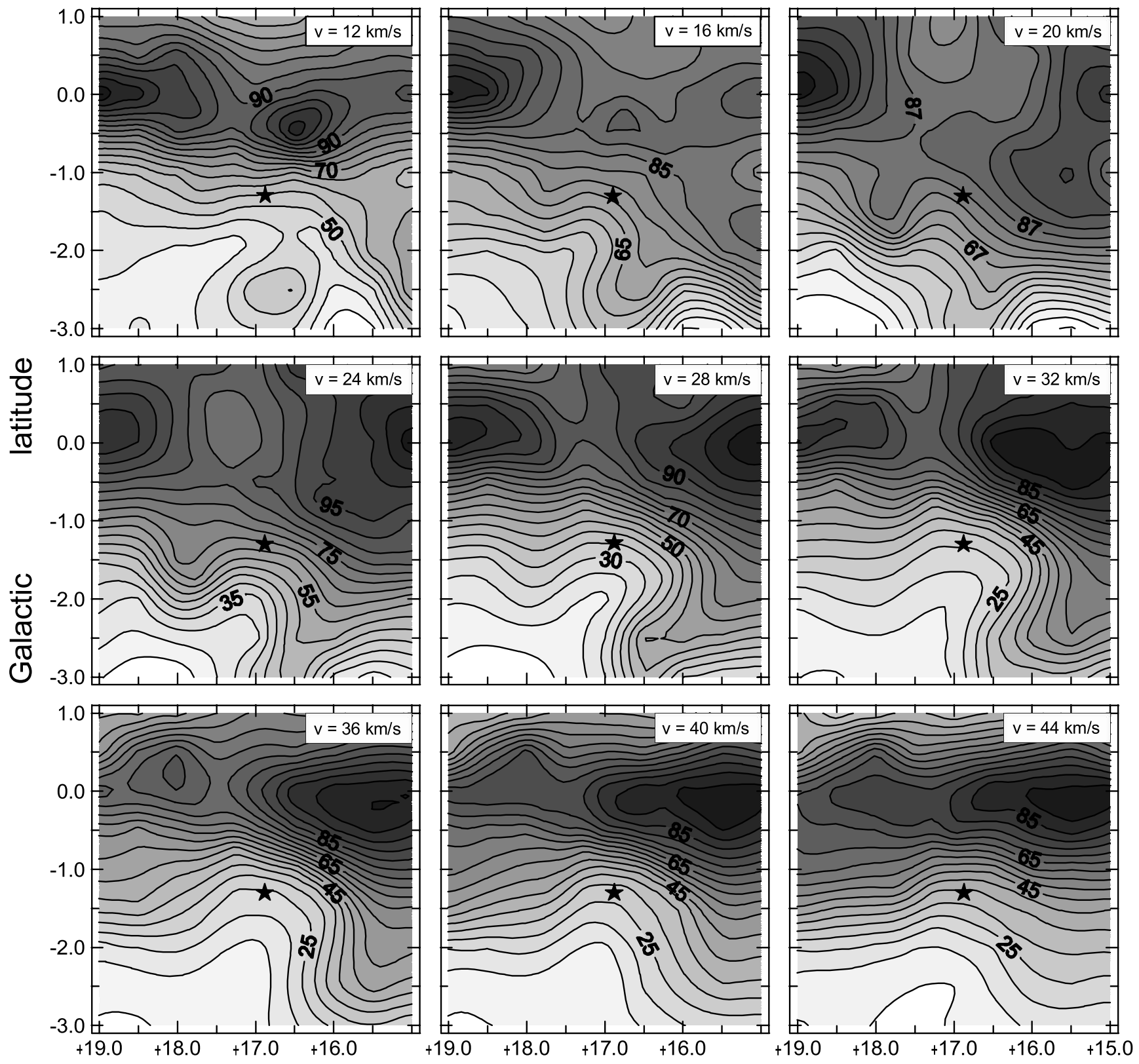

\section{Galactic longitude}

Fig. 5. Neutral hydrogen channel maps for the velocity interval $10-46 \mathrm{~km} \mathrm{~s}^{-1}$, towards LS 5039 . Each map covers $4 \mathrm{~km} \mathrm{~s}{ }^{-1}$, the central velocity is given in each label. A local minimum and an open low density cavity can be clearly seen close to the HMXB. The cavity is open towards the direction opposite to the strongest mass concentrations near the galactic plane, as expected from a wind-blown structure.

found for the dynamical age of the bubble $\left(\sim 3 \times 10^{6} \mathrm{yr}\right)$. Nevertheless, the cavity could be originated by LS 5039 if the pre-SN system was close to the actual position, in such a way that the progenitor of the compact object could have also contributed to the formation of the bubble. In such a case, the X-ray binary would be only about $\sim 2 \times 10^{5}$ yr old, and we would expect to find the radio SNR originated in the SN event with a current surface brightness of $\Sigma_{408} \sim 3 \times 10^{-22} \mathrm{~W} \mathrm{~m}^{-2} \mathrm{~Hz}^{-1} \mathrm{sr}^{-1}$ (Caswell $\&$ Lerche 1979). Filtering techniques of the diffuse galactic emission (e.g., Combi et al. 1998) allow to detect very low brightness SNRs with $\Sigma_{408} \sim 10^{-22} \mathrm{~W} \mathrm{~m}^{-2} \mathrm{~Hz}^{-1} \mathrm{sr}^{-1}$, but nothing has been found in this region except for SNR G016.8-01.1, whose surface brightness at $408 \mathrm{MHz}$ would be $\Sigma_{408} \sim 6 \times 10^{-22} \mathrm{~W} \mathrm{~m}^{-2} \mathrm{~Hz}^{-1} \mathrm{sr}^{-1}$ for a typical spectral index $\alpha=-0.5$. This level of flux is expected at this latitude for a younger SNR, say with $t \sim 10^{5} \mathrm{yr}$, but in such a case the particle density of the medium around SNR G016.8-01.1 should be higher than the estimates given in Sect. 5.2, with values of $n \sim 50 \mathrm{~cm}^{-3}$, in order to confine the SNR to its inferred size at $d \sim 2.9 \mathrm{kpc}$. These densities are 1 order of magnitude higher than those 
derived from the H I observations. However, a clear rejection of SNR G016.8-01.1 cannot be established on this basis alone, due to the flux contamination from the $\mathrm{H}$ II region, which could be responsible for errors as large as $100 \%$ in the flux density estimate of the source. Consequently, a picture where the $\mathrm{HI}$ bubble has been created by both the stellar winds from the binary plus the SN explosion remains as an open possibility.

\section{Discussion}

There are two ways to accelerate a binary system by a supernova explosion: ejection of material from the binary in a symmetric SN or an additional velocity kick, produced by asymmetries in the SN itself. In Nelemans et al. (1999), the authors conclude that there is no need of additional velocity kicks in systems like Cygnus X-1 or GRO J1655-40, both containing black hole candidates as the compact objects, to explain their observed large runaway velocities. On the other hand, from the study of Be/X-ray binaries containing neutron stars, van den Heuvel et al. (2000) conclude that, in order to explain the observed large eccentricities and low space velocities of these systems, a kick in an asymmetric SN explosion scenario is needed. Moreover, the microquasar Circinus X-1, containing a neutron star, seems to have experienced a highly asymmetric SN explosion (Tauris et al. 1999). Unfortunately, the nature of the compact object in LS 5039 still remains unknown. However, we can try to study it in the symmetric SN scenario.

Let $M_{\mathrm{X}}$ and $M_{\mathrm{O}}$ be the masses of the compact object and the optical companion, respectively. Taking into account the stellar evolution tracks by Schaerer et al. (1996), we can assume that $M_{\mathrm{O}} \simeq 40 M_{\odot}$. Considering that it must rotate slower than the breakup speed, and using the mass function $f(m)=0.00103 \pm 0.00020 M_{\odot}$ by McSwain et al. (2001), an upper limit of $M_{\mathrm{X}}<9 M_{\odot}$ is obtained. The minimum mass for the compact object would be that of a neutron star of the Chandrasekar mass, $M_{\mathrm{X}}>1.4 M_{\odot}$. Hence, a total mass of $\sim 41-49 M_{\odot}$ remains in the system.

Since the actual eccentricity of the system is $e=$ $0.41 \pm 0.05$, and has a short orbital period of $P=$ $4.117 \pm 0.011$ days, both parameters are expected to decrease with time due to tidal forces which act to recircularize the orbit. Hence, the eccentricity just after the $\mathrm{SN}$ explosion was $e_{\text {post-SN }}>0.41$. Using the equation $\Delta M=e_{\text {post-SN }}\left(M_{\mathrm{X}}+M_{\mathrm{O}}\right)$ and $M_{\mathrm{X}}=1.4 M_{\odot}$ we find that at least $17 M_{\odot}$ where lost in the $\mathrm{SN}$ explosion in the symmetric case. If we adopt $M_{\mathrm{X}}=9 M_{\odot}$ the minimum mass loss is $20 M_{\odot}$. Moreover, in order to have the system bound after the explosion, i.e., $e_{\text {post-SN }}<1$, the mass loss during the SN event must be less than the remaining mass in the system, i.e., $<49 M_{\odot}$. Hence, the values for the reduced mass of the system, defined as $\mu=1 /\left(1+e_{\text {post-SN }}\right)$, range from 0.5 to 0.7. Using Eq. (5) of Nelemans et al. (1999) with the actual values of $P$ and $e$ we find that the re-circularized period will be $P_{\text {re-circ }} \simeq 3$ days. Since the period of the initial, pre-SN, binary system is given by $P_{\mathrm{i}}=\mu^{2} P_{\mathrm{re}-\text { circ }}$, the orbital period before the SN explosion was in the range 0.8 to 1.5 days. Using Kepler's third law and assuming the latter value to allow the maximum separation possible between the progenitor stars, initial total masses between $60-100 M_{\odot}$ lead to semimajor axis in the range $22-26 R_{\odot}$. This means that the two stars did not evolve separately, but probably through a common envelope phase. Hence, a detailed evolution of the system should be carried out in order to try to compute the initial mass of the progenitor star of the compact object, the maximum age of the system before the SN event, and the mass loss during the SN explosion itself. This kind of study is beyond the scope of this paper.

Nevertheless, we can try to explain the measured $v_{\text {sys }}$ in the context of the symmetric SN explosion scenario using the following equation from Nelemans et al. (1999):

$$
\left(\frac{v_{\mathrm{sys}}}{\mathrm{km} \mathrm{s}^{-1}}\right)=213\left(\frac{\Delta M}{M_{\odot}}\right)\left(\frac{M_{\mathrm{O}}}{M_{\odot}}\right)\left(\frac{P_{\mathrm{re}-\mathrm{circ}}}{\text { day }}\right)^{-\frac{1}{3}}\left(\frac{M_{\mathrm{X}}+M_{\mathrm{O}}}{M_{\odot}}\right)^{-\frac{5}{3}}
$$

Assuming that $M_{\mathrm{O}}=40 M_{\odot}$ and $P_{\text {re-circ }}=3$ days we can find two minimum values of $v_{\text {sys }}$ depending on the adopted mass for the compact object. For $M_{\mathrm{X}}=1.4 M_{\odot}$, and hence $\Delta M>17 M_{\odot}$, we find $v_{\text {sys }} \gtrsim 200 \mathrm{~km} \mathrm{~s}^{-1}$. Similarly, for $M_{\mathrm{X}}=9 M_{\odot}$, and hence $\Delta M>20 M_{\odot}$, we find $v_{\text {sys }} \gtrsim 180 \mathrm{~km} \mathrm{~s}^{-1}$. Our measured recoil velocity is $v_{\text {sys }} \simeq 150 \pm 20 \mathrm{~km} \mathrm{~s}^{-1}$, which would be perfectly reached in the SN explosion scenario, as we have just seen. In fact, the measured value is a little bit lower than the computed ones, which could indicate that part of the eccentricity of the system was reached thanks to a kick in an asymmetric SN explosion. However, we must be cautious with the use of the value for $v_{\text {sys }}$ obtained after Eq. (4), because some effects have been ignored, as pointed out by Nelemans et al. (1999), and also the errors in $e$ and $P_{\text {re-circ }}$ could reduce the computed values. Hence, we can say that both, the high eccentricity measured by McSwain et al. (2001), and the high space velocity reported here, are perfectly compatible, within errors, with a symmetric SN explosion scenario. In other words, the actual eccentricity of the system is naturally explained with the mass loss necessary to explain the measured space (recoil) velocity of LS 5039.

Another common mechanism referred to produce runaway stars is based on dynamical ejection from young open clusters. Numerical simulations (Kiseleva et al. 1998) show that it can explain some moderately high-velocity stars observed in the Galactic disk, but only about $1 \%$ of the stars would be ejected with velocities higher than $30 \mathrm{~km} \mathrm{~s}^{-1}$. Hence, it seems difficult to explain the high observed velocity of LS 5039 as a result of such a mechanism.

It is interesting to note that, contrary to other HMXB with low orbital periods, LS 5039 is still in the circularization process. Using the actual value of $P$, a typical value of $R=10.3 R_{\odot}$ for the radius of the $06.5 \mathrm{~V}$ star, and different formalisms (Claret et al. 1995; Claret \& Cunha 1997) we obtain typical circularization timescales of the order of $(0.5-5) \times 10^{5} \mathrm{yr}$. These values are compatible 
with the system being still in the circularization process, since its formation just in the galactic midplane would take $5 \times 10^{5}$ yr to bring it to the actual position according the integration of its trajectory.

At this point we might ask what is the point of studying runaway microquasars. In the case of LS 5039, if the SN explosion had taken place recently, we could expect the system to survive for the following $\sim 5 \mathrm{Myr}$, because the O6.5 star is still in the main sequence. Hence, according to the computed trajectory, it would reach a height of $Z \simeq-600 \mathrm{pc}$, which translates into $b \simeq-12^{\circ}$. On the other hand, LS 5039 could be associated with the high energy $\gamma$-ray source 3EG J1824-1514, as suggested by Paredes et al. (2000). Hence, if this association is correct, we could be able to detect $\gamma$-ray microquasars up to values of $|b|>10^{\circ}$, although all confirmed microquasars lie within $|b|<5^{\circ}$. In particular, runaway microquasars could be connected with some of the unidentified faint, variable, and soft $\gamma$-ray EGRET sources above the galactic plane, as suggested by Romero (2001) and Mirabel et al. (2001).

Hence, it would be interesting to study the proper motions and radial velocities of as many microquasars as possible. Unfortunately, this kind of information is not easy to obtain for a wide sample of systems. Proper motions can be obtained either from optical data or either from radio data. However, most optical counterparts of these sources are faint objects not present in old astrometric catalogs. Hence, it is mandatory to acquire new positions. In this context, it is better to do it in the radio domain because the uncertainties are always smaller than those obtained in the optical. On the other hand, it is necessary to carry out optical spectroscopy to obtain the radial velocity curve of these sources and use the correct value for the radial velocity of the system.

An alternative approach could be the search for signatures of bow shocks in the microquasar vicinity. However, recent studies by Huthoff \& Kaper (2002) of runaway OB stars, reveal that the success of this method is highly dependant on the density of the ISM around the runaway object. In particular, in only one object, namely Vela X-1, a bow shock has been detected. Finally, one could search for signatures of explosive events in the ISM. Sensitive spectral line observations in the radio are probably the best tool for this purpose, as done in GRO J1655-40 by Combi et al. (2001).

In any case, the study of runaway microquasars such as LS 5039 is likely to contribute significantly to different areas of modern high-energy Astrophysics with independence of the observing technique.

\section{Conclusions}

After an in-depth study of the proper motions and surroundings of LS 5039 our main conclusions are:

1. Positions at optical and radio wavelengths have been used to compute independent optical and radio proper motions, which are perfectly compatible. Therefore, based only on astrometric data we are able to confirm that both, the optical and the radio emission, originate in the same object.

2. From the combined optical and radio positions we have computed an accurate proper motion for LS 5039. This, together with the new estimate of $2.9 \pm 0.3 \mathrm{kpc}$ for the distance, allows us to compute a space velocity of $(U=51, V=-71, W=-118) \mathrm{km} \mathrm{s}^{-1}$ in its Regional Standard of Rest (RSR). This results implies that LS 5039 is a runaway microquasar with $v_{\text {sys }} \simeq 150 \mathrm{~km} \mathrm{~s}^{-1}$, escaping from its own RSR with a large velocity component perpendicular to the galactic plane. This is probably the result of the SN event that created the compact object in this binary system.

3. We have computed the past trajectory of LS 5039. Two $\mathrm{OB}$ associations have been found close to its path in the plane of the sky. However, they are too close to us to be related to the microquasar. On the other hand, we have also found three SNRs near the path of LS 5039. After discarding two of them based on distance arguments, we have focused our attention on SNR G016.8-01.1. A study of this source could not clearly confirm nor reject the association due to the large uncertainties in the estimated radio flux density of the SNR. This fact perhaps justifies future, high sensitivity searches of low-brightness remnants in this region.

4. We have found a semi-open H I cavity close to the LS 5039 position. Although the $\mathrm{O}((\mathrm{f}))$ star in this microquasar seems to be the main agent forming the bubble, a contribution from the progenitor of the compact object cannot be ruled out.

5. Finally, we are able to explain both, the high space velocity and the high eccentricity observed, in a symmetric SN explosion scenario with a mass loss of $\Delta M \sim$ $17 M_{\odot}$.

Acknowledgements. We are grateful to S. E. Urban for kindly providing information from the AC 2000.2 catalog, and for useful discussion on the Tycho-2 proper motion. We are also grateful to D. G. Monet and N. Zacharias for kindly providing information on USNO-A2.0 and UCAC1 catalogs, respectively. M. R. acknowledges useful comments and discussions with I. Ribas, F. Figueras, D. Fernández, J. Colomé and E. Masana, members of Departament d'Astronomia i Meteorologia, at Universitat de Barcelona. This research has made use of the SIMBAD database, operated at CDS, Strasbourg, France. The authors acknowledge the data analysis facilities provided by the Starlink Project which is run by CCLRC on behalf of PPARC. The Guide Star Catalog was produced at the Space Telescope Science Institute under U.S. Government grant. M.R. is supported by a fellowship from CIRIT (Generalitat de Catalunya, Ref. 1999 FI 00199). J.M.P., J.M. and M.R. acknowledge partial support by DGI of the Ministerio de Ciencia y Tecnología (Spain) under grant AYA2001-3092. They also acknowledge partial support by the European Regional Development Fund (ERDF/FEDER). G.E.R. is supported by the research grants PICT 03-04881 (ANPCT) and PIP 0438/98 (CONICET), as well as by Fundación Antorchas. He is very grateful to staff of the Max Planck Institut für Kernphysik at 
Heidelberg, where part of his research for this project was carried out. J.M. has also been aided in this work by an Henri Chrétien International Research Grant administered by the American Astronomical Society.

\section{References}

Ankay, A., Kaper, L., de Bruijne, et al. 2001, A\&A, 370, 170 Benaglia, P., \& Cappa, C. E. 1999, A\&A, 346, 979

Caswell, J. L., \& Lerche, I. 1979, MNRAS, 187, 201

Castro-Tirado, A. J., Greiner, J., \& Paredes, J. M. 2001, Proc. of the Third Microquasar Workshop Galactic Relativistic Jet Sources, ed. A. J. Castro-Tirado, J. Greiner, \& J. M. Paredes (Kluwer Academic Publishers)

Claret, A., \& Cunha, N. C. S. 1997, A\&A, 318, 187

Claret, A., Giménez, A., \& Cunha, N. C. S. 1995, A\&A, 299, 724

Clark, J. S., Reig, P., Goodwin, S. P., et al. 2001, A\&A, 376, 476

Combi, J. A., Romero, G. E., \& Benaglia, P. 1998, A\&A, 333, L91

Combi, J. A., Romero, G. E., Benaglia, P., \& Mirabel, I. F. 2001, A\&A, 370, L5

Condon, J. J., Cotton, W. D., Greisen, E. W., et al. 1998, AJ, 115,1693

Dauphole, B., \& Colin, J. 1995, A\&A, 300, 117

Deutschman, W. A., Davis, R. J., \& Schild, R. E. 1976, ApJS, 30,97

Drilling, J. S. 1991, ApJS, 76, 1033

Dubner, G., Giacani, E., Reynoso, E., et al. 1999, AJ, 118, 930

Fich, M., Blitz, L., \& Stark, A. A. 1989, ApJ, 342, 272

Forbes, D. 2000, AJ, 120, 2594

Green, D. A. 2000, A Catalog of Galactic Supernova Remnants, Mullard Radio Astronomy Observatory (Cambridge, England, UK) (available on the World Wide Web at http://www.mrao.cam.ac.uk/surveys/snrs/)

Hannikainen, D., Campbell-Wilson, D., Hunstead, R., et al. 2001, in Proc. of the Third Microquasar Workshop Galactic Relativistic Jet Sources, ed. A. J. Castro-Tirado, J. Greiner, \& J. M. Paredes (Kluwer Academic Publishers), Ap\&SS, 276, 45

Høg, E., Fabricius, C., Makarov, V. V., et al. 2000, A\&A, 357, 367

Huthoff, F., \& Kaper, L. 2002, A\&A, 383, 999

Johnson, H. L. 1966, ARA\&A, 4, 193

Kaper, L., Camerón, A., \& Barziv, O. 1999, in Proc. of the IAU Symp. 193, Wolf-Rayet phenomena in massive stars and starburst galaxies, ed. K. A. van der Hucht, G. Koenigsberger, \& P. R. J. Eenens, ASP Conf. Ser., 193, 316

Kiseleva, L. G., Colin, J., Dauphole, B., \& Eggleton, P. 1998, MNRAS, 301, 759

Lahulla, J. F., \& Hilton, J. 1992, A\&AS, 94, 265

Lasker, B. M., Sturch, C. R., McLean, B. J., et al. 1990, AJ, 99, 2019

Lejeune, T., \& Schaerer, D. 2001, A\&A, 366, 538

Liu, Q. Z., van Paradijs, J., \& van den Heuvel, E. P. J. 2000, A\&AS, 147, 25

Liu, Q. Z., van Paradijs, J., \& van den Heuvel, E. P. J. 2001, A\&A, 368, 1021

Martí, J., Paredes, J. M., \& Ribó, M. 1998, A\&A, 338, L71

McSwain, M. V., Gies, D. R., Riddle, R. L., Wang, Z., \& Wingert, D. W. 2001, ApJ, 558, L43

Melnik, A. M., \& Efremov, Y. N. 1997, VizieR Online Data Catalog, 902, 110013
Mirabel, I. F., \& Rodríguez, L. F. 1999, ARA\&A, 37, 409

Mirabel, I. F., Dhawan, V., Mignani, R. P., Rodrigues, I., \& Guglielmetti, F. 2001, Nature, 413, 139

Monet, D. G., Bird, A., Canzian, B., et al. 1999, USNO-A2.0 CD-ROM (U.S. Naval Observatory, Washington DC)

Morrison, J. E., Röser, S., McLean, B., Bucciarelli, B., \& Lasker, B. 2001, AJ, 121, 1752

Motch, C., Haberl, F., Dennerl, K., Pakull, M., \& JanotPacheco, E. 1997, A\&A, 323, 853

Nelemans, G., Tauris, T. M., \& van den Heuvel, E. P. J. 1999, A\&A, 352, L87

Ochsenbein, F., Bauer, P., \& Marcout, J. 2000, A\&AS, 143, 23

Paredes, J. M., Martí, J., Ribó, M., \& Massi, M. 2000, Science, 288,2340

Reed, B. C. 2000, AJ, 120, 314

Reich, W., Fuerst, E., Reich, P., Sofue, Y., \& Handa, T. 1986, A\&A, 155, 185

Reynoso, E. M., \& Mangum, J. G. 2000, ApJ, 545, 874

Ribó, M., Reig, P., Martí, J., \& Paredes, J. M. 1999, A\&A, 347,518

Ribó, M., Paredes, J. M., Martí, J., \& Massi, M. 2002, in preparation

Rodgers, A. W., Campbell, C. T., \& Whiteoak, J. B. 1960, MNRAS, 121, 103

Romero, G. E. 2001, in Proc. of The Nature of Unidentified Galactic High-energy Gamma-ray Sources, ed. A. Carramiñana, O. Reimer, \& D. J. Thompson, ASSL Ser. 51 (Kluwer Academic Publishers), 65

Schaerer, D., de Koter, A., Schmutz, W., \& Maeder, A. 1996, A\&A, 312, 475

Schmidt-Kaler, Th. 1982, Landolt-Börnstein, Numerical Data and Functional Relationships in Science and Technology, New Ser. Group 6 Astron. and Astrophys., vol. 2

Sedov, L. I. 1959, Similarity and Dimentional Methods in Mechanics (John Wiley and Sons, New York)

Shahbaz, T., van der Hooft, F., Casares, J., Charles, P. A., \& van Paradijs, J. 1999, MNRAS, 306, 89

Shklovskii, I. S. 1968, Supernovae (John Wiley and Sons, New York)

Spitzer, L. 1998, Physical Processes in the Interstellar Medium (John Wiley and Sons, New York)

Stirling, A. M., Spencer, R. E., de la Force, C. J., et al. 2001, MNRAS, 327, 1273

Tasker, N. J., Condon, J. J., Wright, A. E., \& Griffith, M. R. 1994, AJ, 107, 2115

Tauris, T. M., Fender, R. P., van den Heuvel, E. P. J., Johnston, H. M., \& Wu, K. 1999, MNRAS, 310, 1165

Torra, J., Fernández, D., \& Figueras, F. 2000, A\&A, 359, 82

Urban, S. E., Corbin, T. E., Wycoff, G. L., et al. 1998, AJ, 115,1212

Vacca, W. D., Garmany, C. D., \& Shull, J. M. 1996, ApJ, 460, 914

van den Heuvel, E. P. J., Portegies Zwart, S. F., Bhattacharya, D., \& Kaper, L. 2000, A\&A, 364, 563

van den Heuvel, E. P. J. 1978, in Physics and Astrophysics of Neutron Stars and Black Holes, ed. R. Giacconi, \& R. Ruffini (Amsterdam: North Holland), 828

Zacharias, N., \& Zacharias, M. I. 1999, AJ, 118, 2503

Zacharias, N., Zacharias, M. I., \& de Vegt, C. 1999, AJ, 117, 2895

Zacharias, N., Urban, S. E., Zacharias, M. I., et al. 2000, AJ, 120,2131 